\title{
Comparisons between global and local gyrokinetic simulations of an ASDEX Upgrade H-mode plasma
}

\author{
Alejandro Bañón Navarro, ${ }^{1, \text { a) }}$ Daniel Told, ${ }^{1}$ Frank Jenko, ${ }^{1}$ Tobias Görler, ${ }^{2}$ Tim Happel, ${ }^{2}$ and the ASDEX \\ Upgrade Team $^{2}$ \\ ${ }^{1)}$ Department of Physics and Astronomy, University of California, Los Angeles, California 90095, \\ USA \\ ${ }^{2)}$ Max-Planck-Institut für Plasmaphysik, Boltzmannstrase 2, 85748 Garching, Germany
}

(Dated: 29 March 2017)

\begin{abstract}
We investigate by means of local and global nonlinear gyrokinetic GENE simulations an ASDEX Upgrade H-mode plasma. We find that for the outer core positions (i.e. $\rho_{\text {tor }} \approx 0.5-0.7$ ), nonlocal effects are important. For nominal input parameters local simulations over-predict the experimental heat fluxes by a large factor, while a good agreement is found with global simulations. This was a priori not expected, since the values of $1 / \rho^{\star}$ were large enough that global and local simulations should have been in accordance. Nevertheless, due to the high sensitivity of the heat fluxes with respect to the input parameters, it is still possible to match the heat fluxes in local simulations with the experimental and global results by varying the ion temperature gradient within the experimental uncertainties. In addition to that, once an agreement in the transport quantities between local (flux-matched) and global simulations is achieved, an agreement for other quantities such as density and temperature fluctuations is also found. The case presented here clearly shows that even in the presence of global size-effects, the local simulation approach is still a valid and accurate approach.
\end{abstract}

\section{INTRODUCTION}

It has been known for several decades that in the absence of macroscopic instabilities, turbulent transport is a key limiting factor in the performance of magnetically confined plasmas. In this respect, the gyrokinetic theory ${ }^{1}$ has been shown to be the most adequate framework to describe the microinstabilities which cause the turbulent transport. However, due to its complexity, the gyrokinetic-Maxwell system of equations cannot generally be solved analytically, so that a numerical approach has to be taken.

The emergence of massively parallel computers during the last two decades and the development of gyrokinetic solvers ${ }^{2}$ has opened the road to more and more accurate simulations of magnetized plasmas in realistic conditions. One of the goals of fusion research today is thus, to perform first-principle simulations and make quantitively accurate predictions of experimentally measured quantities over a broad range of plasma conditions.

An important and necessary step towards the achievement of this goal is to demonstrate that the gyrokinetic codes are able to reproduce all of the turbulence features found in current devices. This so-called validation process has lead to quantitatively comparisons between experimental data and results of gyrokinetic simulations by comparing simultaneously several quantities. Here, the improvement in fluctuation diagnostics, which allowed to measure turbulence features, such as density and temperature fluctuations, wavenumber spectra, and even cross-phases between different quantities with high precision, has been indispensable for the validation process. The results from DIII-D ${ }^{3}-13$, Alcator C-Mod ${ }^{14}$, Tore Supra ${ }^{17}$, in FT-2 ${ }^{18}$ and ASDEX Upgrade $\frac{19}{23}$ are examples of such efforts.

a)Electronic mail: banon@ physics.ucla.edu
However, with the exception of a few results $10|11| 19|23| 24$ where global simulations have been used, most of the gyrokinetic validation studies made use of the local approximation. The local (flux-tube) approximation assumes that the relevant turbulent structures are small with respect to the characteristic scales of the profiles and gradients. This allows for the use of periodic boundary conditions and the application of spectral methods, which leads to a reduction in the numerical cost of the simulations with respect to the global simulations.

It has also been shown that local and global simulations agree in the limit where the local approximation is valid $11|1923| 25 \mid 27$, although mainly simple ideal cases have been used. The scarcity of results motivated us to extend the previous work of Refs. [20,22] for an ASDEX Upgrade in the high-confinement regime (H-mode) plasma, by additionally presenting global simulation results and testing the validity of the local approximation by comparing local and global results for several fluctuating quantities.

The paper is organized as follows. In Section II an overview of the plasma discharge analyzed is given. A description of the gyrokinetic simulation method used is described in Section III] Linear gyrokinetic simulations are presented in Section IV. The main results of the paper are shown in Section $\mathrm{V}$. Here, the comparisons between local and global simulations for ion and electron heat fluxes, density and temperature fluctuations are shown. Finally, conclusions and future work will be discussed in Section VI.

\section{OVERVIEW OF THE PLASMA DISCHARGE}

We will study the ASDEX Upgrade discharge \#28245 which has already been investigated in Refs. [20, 22]. The discharge was operated in the $\mathrm{H}$-mode, with a magnetic field strength on axis of $B=2.27 \mathrm{~T}$ and a plasma current of $I_{p}=600 \mathrm{kA}$. The input neutral beam injection (NBI) was $2.5 \mathrm{MW}$ and electron cyclotron resonance heating (ECRH), 

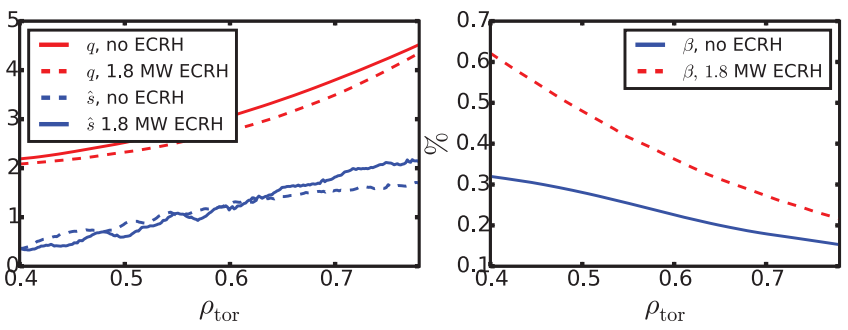

FIG. 1. Global profiles as a function of the normalized toroidal flux radius for the two phases studied in this paper. (Left) Safety factor $(q)$ and magnetic shear $(\hat{s})$. (Right) Plasma beta $(\beta)$.
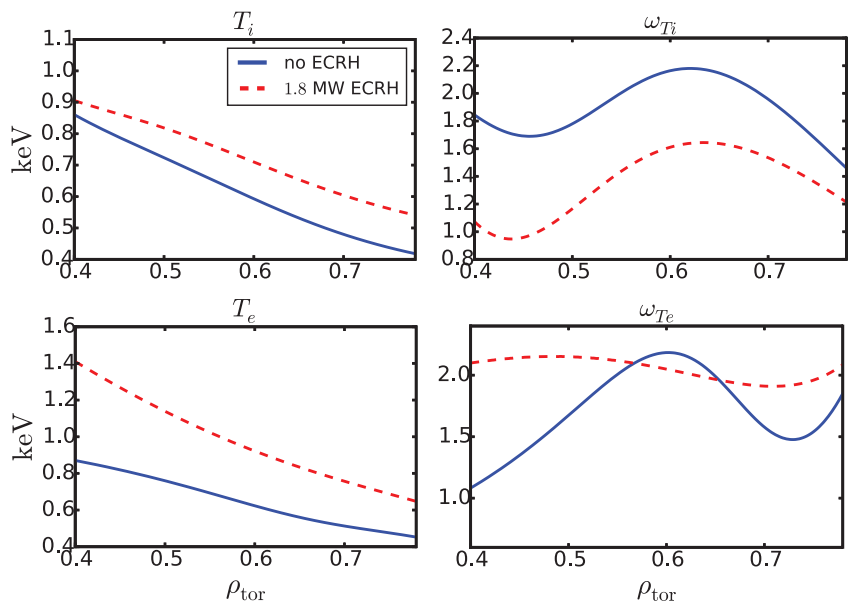

FIG. 2. Ion (top) and electron (bottom) temperature and logarithmic temperature gradients profiles, as a function of the normalized toroidal flux radius for the two phases studied in this paper.

which was divided into four phases, $0.0,0.5,1.2$, and 1.8 $\mathrm{MW}$, respectively, was applied subsequently at intervals of 0.5 seconds. As in Ref. [21], in this work, we will focus on the phases without ECRH and with 1.8 MW ECRH. The main plasma profiles are shown in Figs. 1 and 2 Here, we chose the normalized toroidal flux radius, $\rho_{\text {tor }}=\sqrt{\Phi_{\text {tor }} / \Phi_{\text {edge }}}$, as the radial coordinate, where $\Phi_{\text {tor }}$ is the toroidal magnetic flux and $\Phi_{\text {edge }}$ is its value at the last closed flux surface. Finally, the logarithmic gradients are defined as $\omega_{X}=-\frac{1}{X} \frac{d X}{d \rho_{\text {tor }}}$, with $X$ being the ion $\left(T_{i}\right)$ and the electron $\left(T_{e}\right)$ temperature, respectively.

\section{THE GYROKINETIC METHOD}

The turbulence data obtained in this paper is produced with the gyrokinetic code GENE ${ }^{28}$, which solves self-consistently the gyrokinetic-Maxwell system of equations on a fixed grid in five dimensional phase space (plus time): three magnetic field-aligned coordinates $x, y, z$ and two velocity coordinates $v_{\|}, \mu$, with radial $(x)$, binormal $(y)$, parallel $(z)$, parallel velocity $\left(v_{\|}\right)$, and magnetic moment $(\mu)$, respectively. GENE has the possibility of simulating either a flux-tube ${ }^{29}$ (local simulations) or full surface (global simulations). In local sim- ulations, it is assumed that the relevant turbulent structures are small with respect to the radial variation of the background profiles and gradients. This allows us to use periodic boundary conditions and thus, the coordinates perpendicular to the magnetic field are Fourier transformed $(x, y) \rightarrow\left(k_{x}, k_{y}\right)$. The flux-tube approach is generally a good approximation when $\rho^{\star}=\rho_{s} / a \rightarrow 0$, where $\rho^{\star}$ is a normalized gyroradius, $\rho_{s}=c_{s} / \Omega_{i}$ is a reference gyroradius defined with the ion sound speed $c_{s}=\sqrt{T_{e} / m_{i}}$ and the ion gyrofrequency $\Omega_{i}=q_{i} B_{0} /\left(m_{i} c\right)$, and $a$ is the minor radius of the tokamak. Here, $m_{i}$ denotes the ion mass, $q_{i}$ its charge, $T_{e}$ the electron temperature, $B_{0}$ a reference magnetic field and $c$ the speed of light. In contrast, in global simulations, the radial dependence of the profiles is kept and Dirichlet or Von Neumann boundary conditions and buffer zones are required in the radial direction. The values of the inverse normalized gyroradius $\left(1 / \rho^{\star}\right)$ for the cases analyzed in this paper are shown in Fig. 3 According to literature $11|1923| 25 \mid 27$, these values are large enough such that local and global results should be in agreement. However, we anticipate now that global effects are indeed important for this discharge.

There are two ways of running global simulations. The so called flux-driven approach 27/30/32, in which input sources of energy are applied in order to mimic the heating by the external source of the experiments, and the gradient-driven approach 33 35. which tries to maintain the distribution function near its initial value, so that the gradients and the turbulence drive are approximately constant during the simulation. This is achieved by adding Krook-type particle and heat sources and sinks to the right-hand side of the gyrokinetic equation. The former approach is the most physically relevant. However, it has some drawbacks. On the one hand, modeling the external sources is very challenging from a numerical perspective. On the other hand, as the profiles are not known a priori, the simulations must be run over confinement time scales rather than turbulence time scales, as it is in the case for local simulations. In this work, the gradient-driven method is adopted. These simulations are still much more challenging to run than local simulations for several reasons. They are more expensive computationally and there are also more parameters to set up, making convergence studies much more demanding.

The detailed implementation of the additional parameters required for global gradient-driven simulations for GENE can be found in Refs. [35|33|27]. In the following, as a reference, we summarize the main specific global parameters that have been used in this work, and from which convergence results have been achieved. Other parameters, such as resolution, box sizes, etc., which are common to both local and global simulations are given in the following sections. First, Krook particle and heat source (and sink) terms with coefficients between $0.02-0.2 c_{s} / a$ have been used. The application of the Krook sources is smoothed using a Gaussian filter in the radial direction to prevent the Krook sources from acting on very small scales (filter widths of 10 and $25 \%$ of the radial domain have been tested). Moreover, Dirichlet boundary conditions are considered in the radial direction, where upper and lower buffer zones between $5-10 \%$ have also been checked. 


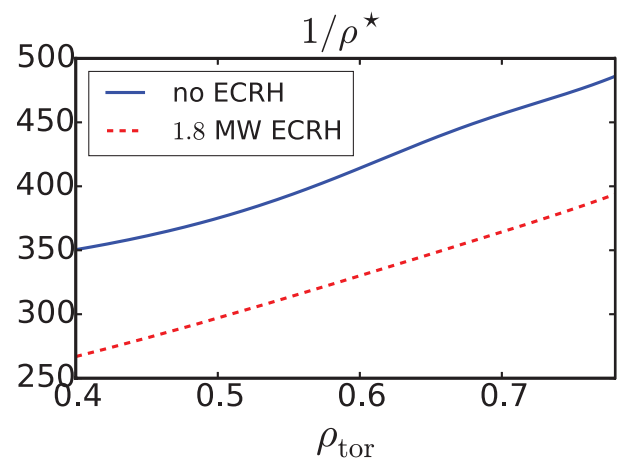

FIG. 3. Profile of the inverse normalized gyroradius $\left(1 / \rho^{\star}\right)$ for the phases without and with 1.8 MW ECRH.

Here, an artificial Krook damping operator is applied in the buffer regions (value of $1.0 c_{s} / a$ has been used) in order to avoid unphysical profile variations close to the boundaries.

\section{LINEAR GYROKINETIC SIMULATIONS}

In this section, we compare the linear growth rates obtained from global simulations versus the linear growth rates from local simulations. For both cases, the following features of the GENE code were used: two particle species (deuterons and electrons), electromagnetic effects by solving the parallel component of Ampère's law, and a linearized LandauBoltzmann collision operator with energy and momentum conserving terms ${ }^{36}$, which includes also the effective charge (with $Z_{\mathrm{eff}}=1.2$ ) in order to mimic the effect of collisionality of the missing impurities (mainly Boron) in two kinetic species simulations. Linear and nonlinear flux-tube simulations with Boron as a third kinetic species were carried out, but differences of less than $10 \%$ with respect to the two kinetic species cases were found for growth rates and heat fluxes. As a result, global simulations with three kinetic species were not performed. The magnetic equilibrium geometry is taken from the TRACER-EFIT interface ${ }^{37}$. Finally, hyperdiffusion terms are used to stabilize spurious grid-size oscillation $\mathbf{3}^{38}$.

The radial domain used in global simulations extends from $\rho_{\text {tor }}=0.31-0.78$ for the case without ECRH and from $\rho_{\text {tor }}=0.40-0.80$ for the case with ECRH. The growth rates for the local simulations are calculated by maximizing the local growth rates over the global radial domain and the ballooning angles in order to take into account the eddy tilting in the global simulations. As in Ref. [21], in the following, we will focus on the low wave-number range where the dominant instability is an ion temperature gradient (ITG) mode. Fig. 4 shows the results for the growth rates. It is observed that the growth rates of the global simulations are below the local values for all toroidal numbers, as one should expect. Moreover, the maximum growth rate of global simulations is around $40 \%$ lower than the maximum growth rate for the local simulations. This difference could imply the existence of global effects in these simulations, as we will see in the following section.

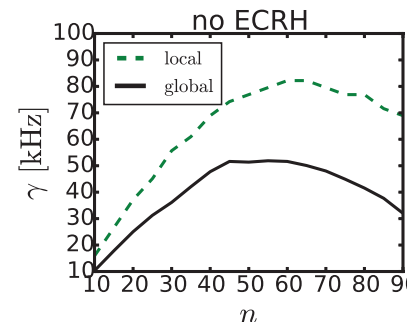

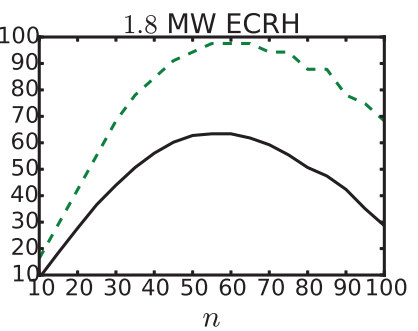

FIG. 4. Linear growth rates for local and global simulations versus the toroidal mode $(n)$ for the two different heating scenarios. In this range, the main instability is an ion temperature gradient (ITG) mode.

\begin{tabular}{|l|c|c|}
\hline Parameter & Local & Global \\
\hline \hline Radial domain $\left(\rho_{\text {tor }}\right)$ & $0.47,0.56,0.67$ & $(0.31-0.78)$ and $(0.40-0.80)$ \\
\hline Spatial Resolution $(x \times y \times z)$ & $256 \times 128 \times 32$ & $512 \times 128 \times 32$ \\
\hline Velocity resolution $\left(v_{\|} \times \mu\right)$ & $48 \times 16$ & $64 \times 48$ \\
\hline Radial box size $(x)$ & $125 \rho_{s}$ & $187 \rho_{s}$ \\
\hline Binormal box size $(y)$ & $125 \rho_{s}$ & $157 \rho_{s}$ \\
\hline Parallel velocity domain per species $j$ & $0-3 v_{T j}$ & $0-3.9 v_{T j}$ \\
\hline Magnetic moment domain per species $j$ & $0-9 T_{j} / B_{0}$ & $0-15 T_{j} / B_{0}$ \\
\hline
\end{tabular}

TABLE I. Local and global parameters. Here, $T_{j}$ is the background temperature of species $j$ taken at the center of the domain, $v_{T j}=$ $\sqrt{2 T_{j} / m_{j}}$ is the thermal velocity of species $j$ and $B_{0}$ is the magnetic field at the center of the domain.

\section{NONLINEAR GYROKINETIC SIMULATIONS}

In addition to the features used in linear simulations, external $E \times B$ shear and parallel flow shears are also included in nonlinear simulations, and the results of the simulations are time-averaged over a range well exceeding the correlation time of the underlying turbulence (approximately $500 c_{s} / a$ time units). A comparison between the requirements of local and global simulations are given in Table [ Global simulations required at least 2 times more spatial resolution than local box size due to the larger box size. Also, due to the difference in temperature between the inner and the outer core regions, global simulations also require both a larger velocity domain and higher velocity resolution. This adds another factor of 4 in velocity resolution. However, in local simulations, in order to obtain a profile, one needs to do several local simulations. In this work, we have performed 3 local simulations for each phase. This makes the computational cost of global simulations at least a factor of 2 more expensive than the local ones. However, as we mentioned previously, convergence studies for global simulations are more demanding than for local simulations, and most of the computational time of the global simulations is spent in performing convergence studies, making global simulations roughly an order of magnitude more expensive than the local simulations.

\section{A. Turbulent ion and electron heat fluxes}

In the following, we compare the experimental ion and electron heat fluxes obtained through power balance analysis with 

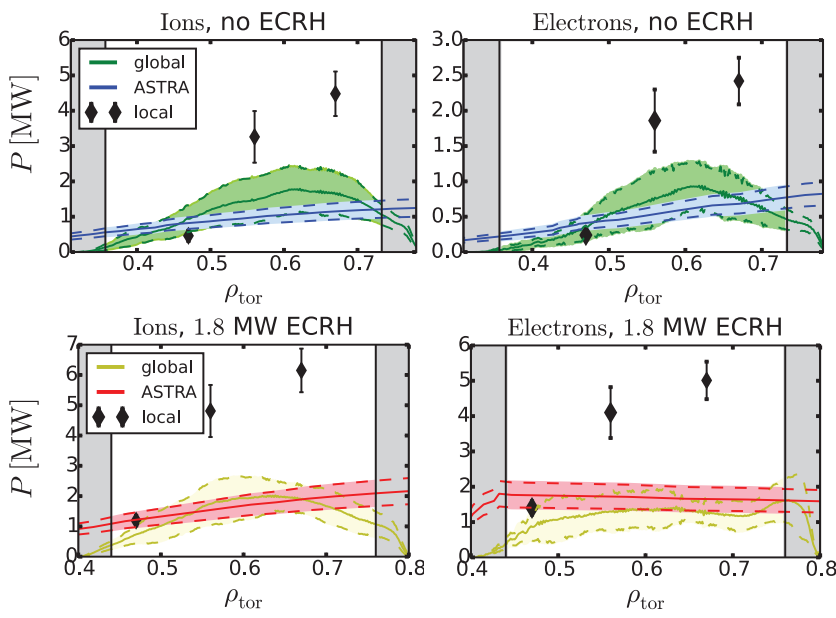

FIG. 5. (Color online) Radial profiles of the heat fluxes for top) phase without ECRH and bottom) phase with 1.8 MW for both ions (left) and electrons (right). Here, the simulations use the nominal (experimental) profiles.

The power balance analysis with ASTRA has been presented in Ref. 20.

the ASTRA ${ }^{39}$ code and the results from local and global nonlinear GENE simulations. The results are shown in Fig. 5 . Here, the ion (electron) heat fluxes are shown on the left (right) for both phases. The solid blue (red) lines indicate the ASTRA results and their shaded regions are used to indicate the uncertainty of the ASTRA values. Although for this discharge, we do not have a rigorous estimate of the uncertainty of the ASTRA results, a $20 \%$ uncertainty has been chosen as an approximate value. The markers represent the local gyrokinetic simulations. Global simulation results are shown in solid green (yellow) lines, and their shaded region correspond to their standard deviation around the mean value. Finally, in the shaded-gray areas, the buffer zones $(10 \%)$ for the global simulations are marked.

Several conclusions can be drawn from Fig. 5 For the position at $0.46 \rho_{\text {tor }}$, there is good agreement between local GENE and ASTRA results. However, for the outer positions $\{0.56,0.67\} \rho_{\text {tor }}$, local simulations over-predict the ASTRA results by a large factor (3 to 4 times). On the contrary, global simulations are in reasonably good agreement with ASTRA results. In particular, by taking into account their error bars, both global and ASTRA results are practically compatible in all the radial domain within the buffer zones. Note that results inside the buffer zone should not be taken into consideration, since the buffer zone is a transition region, where the heat fluxes are gradually reduced until vanishing at the boundaries.

The large difference ( 3 to 4 times) found between local and global results was unexpected, especially since their growth rates differed only by $40 \%$, and also because of the large values of the inverse normalized gyroradius found in this discharge (see Fig. 3). Moreover, $1 / \rho^{\star}$ is smaller at the inner core (values in the range of $250-350$ ) than at the outer core (around $350-500$ ). Therefore, one should expect (any) differences to be larger in the inner core. However, it is at $\rho_{\text {tor }}=0.46$, where the agreement between global and local

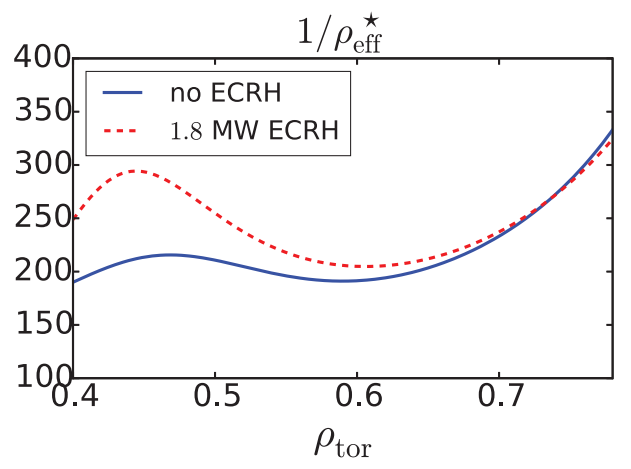

FIG. 6. Profile of the inverse effective normalized gyroradius $\left(1 / \rho_{\text {eff }}^{\star}=L_{T i} / \rho_{i}\right)$ for the phases without and with ECRH.

simulations is better. It is discussed in Ref. [26] that the standard $\rho^{\star}$ parameter may not be the most adequate in determining if the local approximation is valid, when the driving region is narrower than the tokamak minor radius $(a)$. In that work, using a simulation setup with a localized turbulent drive in the radial domain, they defined an effective $\rho_{\text {eff }}^{\star}=\rho^{\star} / \Delta_{r}$, where $\Delta_{r}$ is defined as the full width at half maximum of the gradient profile. However, given the profiles presented in this work, it is not possible to apply $\Delta_{r}$ as they do. For this reason, instead of using $\Delta_{r}$, we used $L_{T i}$, i.e., the ion temperature gradient scale length, to define an effective $\rho^{\star}$. The results are shown in Fig. 6 (right). We observe that now the values (around 200) are reduced with respect to the standard $\rho^{\star}$ definition. Nevertheless, the values are still large enough, that it is a priori not obvious that this alone can be used to judge if the local approximation is valid. This is especially true, independently of the $\rho^{\star}$ definition adopted, for medium size tokamaks such as ASDEX Upgrade or DIII-D, where $1 / \rho^{\star}$ ranges typically from a few to several hundreds. For these cases, it seems that only by performing nonlinear global simulations and comparing to local ones, one can find out if global effects are important. In this regard, it is worth mentioning that ion and electron heat fluxes exhibit radially propagating fronts or avalanches with similar properties (velocity and radial extent) in both local and global simulations. Similar results have been previously reported in Refs. [27|23].

It is also important to note that it is still possible to match the local simulations with the ASTRA (and global) results by varying the experimental input parameters within their experimental uncertainties. In particular, the so called fluxmatched simulations can be achieved at the outer positions $\{0.56,0.67\} \rho_{\text {tor }}$ by decreasing the nominal ion temperature gradient by $20 \%\left(\delta \omega_{T i}=-20 \%\right)$ for the case without ECRH and up to a $30 \%\left(\delta \omega_{T i}=-30 \%\right)$ for the case with $1.8 \mathrm{MW}$ ECRH. These changes in $\delta \omega_{T i}= \pm 20 / 30 \%$, as shown in Ref. [20], are within the estimated uncertainties of the profile fitting routine. The results are shown in Fig.7. Here, although the mean values do not necessarily match perfectly, we can say that within their error bars, local, global and ASTRA results are compatible. Therefore, this discharge is a clear example where small variations in the ion temperature gradients can produce large variations in the heat fluxes. This is interesting 

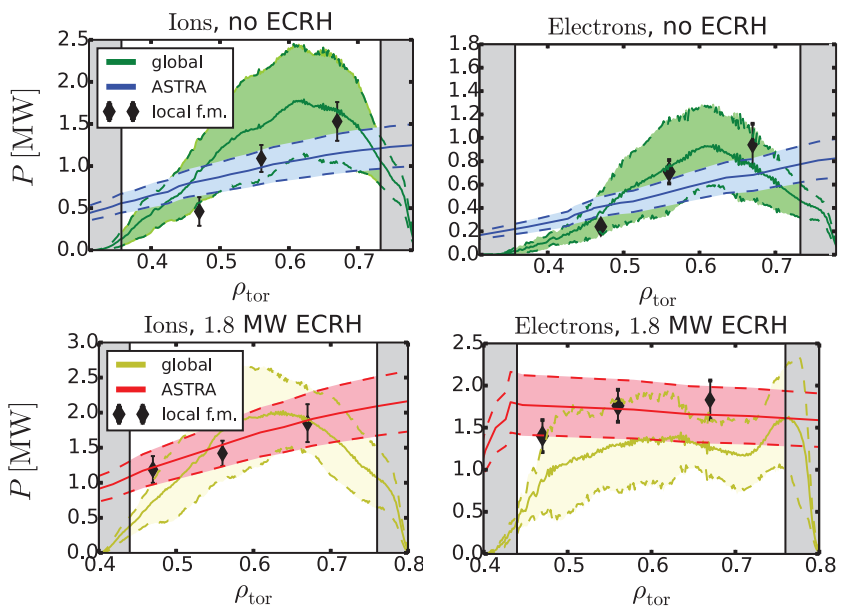

FIG. 7. (Color online) Radial profiles of heat fluxes for top) phase without ECRH and bottom) phase with 1.8 MW for both ions (left) and electrons (right). Here, flux-matched (f.m.) local simulations are compared with global and ASTRA results.
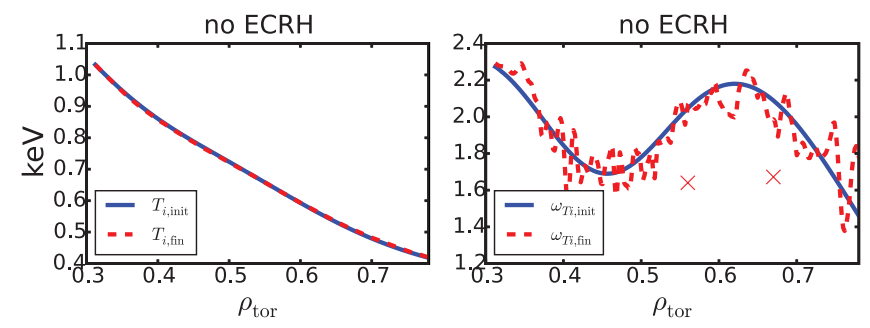

FIG. 8. Variation of the profiles for the case without ECRH. The final profiles are time-averaged over the saturated state of the simulation. The red crosses represent the variation of the nominal logarithmic ion temperature gradient done in the flux-matched simulations.

because, as it was mentioned previously, in global gradientdriven simulations, Krook-type terms are used to keep the profiles and their drive close to their initial values. For this reason, we have also checked that the initial and the timeaveraged final profiles are indeed maintained on average. The results for the ion temperature and its gradient are shown in Fig. 8 for the case without ECRH. As it is observed in the figure, the profiles between the initial and final state are practically indistinguishable from each other. Nevertheless, there are differences in the ion temperature gradient due to the presence of corrugations. However, these corrugations lead to variations in the gradients of a few percent $(\lesssim 5 \%)$, which is still much lower than the $20 \%$ variation needed to achieve local flux-matched results (marked with red crosses in Fig. 8].

To summarize the main results of this section, we found that for the phases studied in this paper, global effects were important. This could not be explained by a relaxation of the profiles in the global simulations and it was not anticipated by the values of $\rho^{\star}$. Nevertheless, it was still possible to match the local results by varying the input parameters within their experimental error bars, so that an agreement between local, global and ASTRA was achieved.

\section{B. Turbulent fluctuation amplitudes}

Having found an agreement for the heat fluxes between flux-matched local and global simulations, it is also important to check if there is also an agreement for other quantities such as density or temperature fluctuations. For instance, in Ref. [11], they found an "unexpected breaking of correlation between fluctuations and transport levels". In particular, local simulations predicted higher electron temperature fluctuations than the global ones, but lower electron heat flux values. Moreover, they also compared both approaches with electron temperature fluctuations measurements, and it was found that global simulations were in better agreement to the experimental results than the local ones. This point is relevant because although the heat fluxes found in both approaches were considered satisfactory, global simulations were necessary to better match the electron temperature fluctuations measurements.

In order to investigate whether similar effects are observed in this discharge, detailed comparisons between flux-matched local and global simulation results for density and ion and electron temperature fluctuations are presented in the following section. We summarize first how the comparisons have been performed, since in GENE, the local and global approaches use a different radial coordinate (wave-number $k_{x}$ for local and $x$ for global). For a given fluctuation quantity $\tilde{A}$, we compare them as follow:

$$
\begin{aligned}
\tilde{A}_{\text {Local }} & =\sqrt{\left\langle\sum_{k_{x} k_{y}}\left|\tilde{A}\left(k_{x}, k_{y}, z=0, t\right)\right|^{2}\right\rangle_{t}}, \\
\tilde{A}(x)_{\text {Global }} & =\sqrt{\left\langle\sum_{k_{y}}\left|\tilde{A}\left(x, k_{y}, z=0, t\right)\right|^{2}\right\rangle_{t}} .
\end{aligned}
$$

Here, $\langle\cdot\rangle_{t}$ represents the time average. Although in this section, we are not comparing simulations with experimental results, we will consider the fluctuations at the outboard midplane (represented by $z=0$ in GENE), since it is close to the area where diagnostics such as Doppler Reflectometry or Correlation Electron Cyclotron Emission (CECE) usually measure.

The results for density, ion and electron temperature fluctuations are shown in Fig. 9 A first glance at the results shows that the agreement between flux-matched local and global simulations is generally good. However, differences between the quantities (and cases) are still present. For instance, while the agreement is very good for the electron temperature fluctuations for the case without ECRH (Fig. 96)), local density fluctuations for the same phase tend to underpredict the global results (Fig. 9(a)).

Analyzing the results in detail, the following conclusions can be drawn. First, for the density fluctuations (Figs. 9 (a) and (b)), we observed that local and global simulations show the same trend with ECRH, i.e., for both cases, the case without ECRH produces more density fluctuations than the case with 1.8 MW ECRH. This is interesting since as it was mentioned in Ref. 20, density fluctuations measured with a Doppler reflectometer for this discharge showed the 

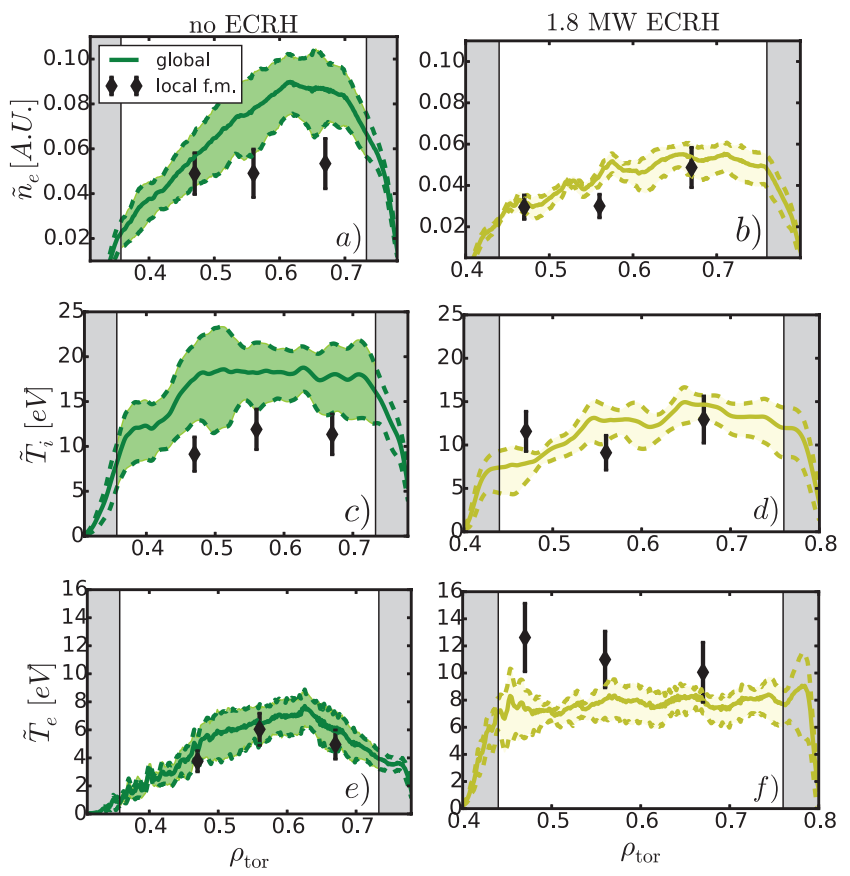

FIG. 9. Electron density (top), ion (middle) and electron temperature (bottom) fluctuation amplitudes. Markers represent the flux-matched (f.m.) local simulations. Global results with the standard deviation around the mean value are shown with solid lines.

opposite trend (i.e., more fluctuation with ECRH). We can now discard global effects as the cause for the disagreement. The radial density profiles also present differences between both approaches, especially for the case without ECRH. For this phase, the local fluctuations do not increase with radius as much as the global ones. Indeed, although the value at $\rho_{\text {tor }}=0.46$ is in good agreement between both approaches, the results for the outer positions of the local simulations are below the results of the global ones. However, these differences are not present for the case with 1.8 MW ECRH.

For the ion temperature fluctuations (Figs. 9 (c) and (d)), similar conclusions can be made. There is a good agreement between local and global simulations for the case with 1.8 MW ECRH. However, for the case without ECRH, local fluctuation amplitudes are below the global ones for all radial positions. Finally, for the electron temperature fluctuations (Figs. 9 (e) and (f)), the agreement is now better for the case without ECRH, while for the case with ECRH, local fluctuations are higher than the global ones. For this case, this difference is consistent with the fact that the mean value of electron heat flux in local simulations is higher than in global ones (Fig. 7).

In order to investigate the correlation between transport levels and fluctuations, the ratio of temperature to heat flux was suggested in Ref. [11] as a measure for such correlation. The ratios for ions (left) and electrons (right) are shown in Fig. 10 , where for simplicity, only the mean values of the quantities have been taken to evaluate the ratios. From this figure, it is clearly observed that there is a very good agreement between flux-matched local and global simulation results. In partic-
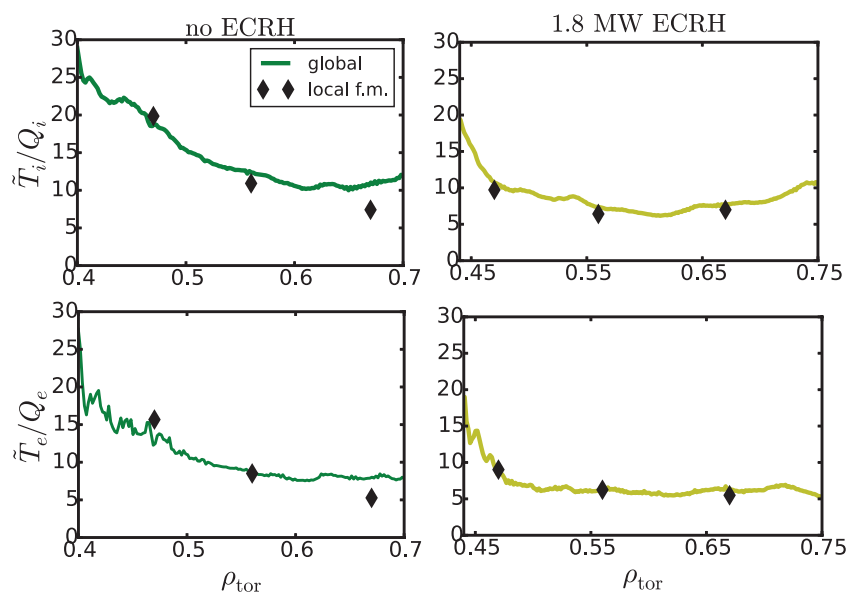

FIG. 10. Comparison of the temperature of the ratio of $\tilde{T}_{i} / Q_{i}$ (left) and $\tilde{T}_{e} / Q_{e}$ (right) for the two phases. Global results are shown with solid lines and flux-matched (f.m.) local results with markers.

ular, both the radial trends and the values coincide almost perfectly between both approaches, especially in the phase with 1.8 MW ECRH. Assuming the same cross-phases between local and global simulations, these results indicate that if the heat fluxes would be perfectly matched between local and global simulations, temperature fluctuations would also be matched for this discharge. Therefore, even in the presence of global size effects, since it is possible to match global and local simulations for both transport and fluctuating quantities, the local simulation approach is still a valid and accurate approach, which can be used for comparisons with experimental measurements and validations of the gyrokinetic model at different levels by comparing simultaneously several quantities.

\section{CONCLUSIONS}

In this paper, we have compared global gradient-driven and local gyrokinetic simulations for an ASDEX Upgrade $\mathrm{H}$-mode plasma using the GENE code. The main results of the paper can be summarized as follows. Local simulations at nominal input parameters at outer core positions $\left(0.56-0.67 \rho_{\text {tor }}\right)$ over-predict by a large factor (3 to 4 times) the ion and electron heat fluxes calculated from power balance analysis with the ASTRA code for the two heating scenarios studied. On the contrary, global gradient-driven simulation results (using the same input profiles as the local ones) were compatible with ASTRA results for practically the whole radial domain.

The large difference found between local and global results at these positions was not expected, since the values of the inverse normalized gyroradius $\left(1 / \rho^{\star} \approx 300-500\right)$ were in the range for which according to previous results global and local simulations should have agreed. In order to better determine when the local approximation is still valid, we introduced an effective $\rho^{\star}$, defined as $\rho_{\text {eff }}^{\star}=\rho_{i} / L_{T i}$, i.e., using the ion temperature gradient scale length instead of the minor radius of 
the tokamak. With this new definition, the values of $1 / \rho_{\text {eff }}^{\star}$ were lower (around 200). In any case, independently of the $\rho^{\star}$ definition adopted, for medium size tokamaks such as ASDEX Upgrade or DIII-D, where $\rho^{\star}$ values range from a few to several hundred, it seems that it is difficult to predict if the local approximation is valid.

In spite of the global size effects present in the simulations, it was still possible to match the local simulations with the experimental heat fluxes by decreasing the logarithmic ion temperature gradient within the experimental error bars $\left(\delta \omega_{T i}=-20 / 30 \%\right)$, so that an agreement between local, global and ASTRA results was found. This high sensitivity of the heat fluxes with respect to the ion temperature gradient found in local simulations, motivated us to check whether the time averaged profiles in the global simulations were kept close to their initial values. Indeed, a relaxation of the profiles could have explained the differences found between global and local simulations. However, this was not the case, and the profiles of global simulations were well maintained on average.

Finally, we compared flux-matched local with global simulations for density and temperature fluctuations. Here, we found in general a reasonably good agreement between both methods, indicating a good correlation between the agreement found in the transport levels with the one present in the fluctuating quantities.

For future work, GENE and ASDEX Upgrade comparisons will continue, aiming to validate the gyrokinetic model at different levels by analyzing simultaneously as many quantities as possible. In particular, comparing the results of density fluctuations and spectra measured with a steerable Doppler reflectometer in ASDEX Upgrade, electron temperature fluctuations measured with a CECE diagnostic recently installed in ASDEX Upgrade and the cross-phases between these quantities, with the results of both local and global simulations. In this regard, the further development of Doppler synthetic diagnostics would be desirable. For instance, with the coupling of full-wave simulations with turbulence data generated by gyrokinetic simulations as performed in Ref. [40] with GYRO for DIII-D or recently with local GENE simulations in Ref. [22] for ASDEX Upgrade.

\section{ACKNOWLEDGMENTS}

We would like to thank Jeremie Abiteboul, Gabriele Merlo and David Hatch for fruitful discussion on the global simulations. We also acknowledge Emiliano Fable for providing the ASTRA data and Paul Crandall for his comments that led to a clearer presentation of the manuscript. The research leading to these results has received funding from the European Research Council under the European Unions Seventh Framework Programme (FP7/2007V2013)/ERC Grant Agreement No. 277870. The gyrokinetic simulations presented in this work used resources of the National Energy Research Scientific Computing Center, a DOE Office of Science User Facility supported by the Office of Science of the U.S. Department of Energy under Contract No. DE-AC02-05CH11231.
${ }^{1}$ A. J. Brizard and T. S. Hahm. Foundations of nonlinear gyrokinetic theory. Rev. Mod. Phys., 79:421-468, Apr 2007.

${ }^{2}$ X. Garbet, Y. Idomura, L. Villard, and T.H. Watanabe. Gyrokinetic simulations of turbulent transport. Nuclear Fusion, 50(4):043002, 2010.

${ }^{3}$ A. E. White, L. Schmitz, G. R. McKee, C. Holland, W. A. Peebles, T. A. Carter, M. W. Shafer, M. E. Austin, K. H. Burrell, J. Candy, J. C. DeBoo, E. J. Doyle, M. A. Makowski, R. Prater, T. L. Rhodes, G. M. Staebler, G. R. Tynan, R. E. Waltz, and G. Wang. Phys. Plasmas, 17(056116), 2008.

${ }^{4}$ C. Holland, A. E. White, G. R. McKee, M. W. Shafer, J. Candy, R. E. Waltz, L. Schmitz, and G. R. Tynan. Phys. Plasmas, 16, 0523012009.

${ }^{5}$ A. E. White, W. A. Peebles, T. L. Rhodes, C. H. Holland, G. Wang, L. Schmitz, T. A. Carter, J. C. Hillesheim, E. J. Doyle, L. Zeng, G. R. McKee, G. M. Staebler, R. E. Waltz, J. C. DeBoo, C. C. Petty, and K. H. Burrell. Measurements of the cross-phase angle between density and electron temperature fluctuations and comparison with gyrokinetic simulationsa). Physics of Plasmas, 17(056103), 2010.

${ }^{6}$ J. C. DeBoo, C. Holland, T. L. Rhodes, L. Schmitz, G. Wang, A. E. White, M. E. Austin, E. J. Doyle, J. C. Hillesheim, W. A. Peebles, Z. Yang C. C. Petty, and L. Zeng. Phys. Plasmas, 17(056105), 2010.

${ }^{7}$ C. Holland, L. Schmitz, T. L. Rhodes, W. A. Peebles, J. C. Hillesheim, G. Wang, L. Zeng, E. J. Doyle, S. P. Smith, R. Prater, K. H. Burrell, J. Candy, R. E. Waltz, J. E. Kinsey, G. M. Staebler, J. C. DeBoo, C. C. Petty, G. R. McKee, Z. Yan, and A. E. White. Phys. Plasmas, 18(056113), 2011.

${ }^{8}$ T.L. Rhodes, C. Holland, S.P. Smith, A.E. White, K.H. Burrell, J. Candy, J.C. DeBoo, E.J. Doyle, J.C. Hillesheim, J.E. Kinsey, G.R. McKee, D. Mikkelsen, W.A. Peebles, C.C. Petty, R. Prater, S. Parker, Y. Chen, L. Schmitz, G.M. Staebler, R.E. Waltz, G. Wang, Z. Yan, and L. Zeng. L-mode validation studies of gyrokinetic turbulence simulations via multiscale and multifield turbulence measurements on the diii-d tokamak. $\mathrm{Nu}$ clear Fusion, 51(6):063022, 2011.

${ }^{9}$ M. W. Shafer, R. J. Fonck, G. R. McKee, C. Holland, A. E. White, and D. J. Schlossberg. Phys. Plasmas, 19, 0325042012.

${ }^{10}$ C. Holland, C.C. Petty, L. Schmitz, K.H. Burrell, G.R. McKee, T.L. Rhodes, and J. Candy. Progress in gyro validation studies of diii-d h-mode plasmas. Nuclear Fusion, 52(11):114007, 2012.

${ }^{11}$ C. Holland, J. C. DeBoo, T. L. Rhodes, L. Schmitz, J. C. Hillesheim, G. Wang, A. E. White, M. E. Austin, E. J. Doyle, W. A. Peebles, C. C. Petty, L. Zeng, and J. Candy. Nuclear Fusion, 52(063028), 2012.

${ }^{12}$ C. Holland, J. E. Kinsey, J. C. DeBoo, K. H. Burrell, T. C. Luce, S. P. Smith, C. C. Petty, A. E. White, T. L. Rhodes, L. Schmitz, E. J. Doyle, J. C. Hillesheim, G. R. McKee6, Z. Yan, G. Wang, L. Zeng, B. A. Grierson, A. Marinoni, P. Mantica, P. B. Snyder, R. E. Waltz, G. M. Staebler, and J. Candy. Nuclear Fusion, 53(083027), 2013.

${ }^{13}$ T. Görler, A. E. White, D. Told, F. Jenko, C. Holland, and T. L. Rhodes. Phys. Plasmas, 21(122307), 2014.

${ }^{14}$ L Lin, M Porkolab, E M Edlund, J C Rost, M Greenwald, N Tsujii, J Candy, R E Waltz, and D R Mikkelsen. Studies of turbulence and transport in alcator c-mod ohmic plasmas with phase contrast imaging and comparisons with gyrokinetic simulations. Plasma Physics and Controlled Fusion, 51(6):065006, 2009.

${ }^{15}$ A. E. White, N. T. Howard, M. Greenwald, M. L. Reinke, C. Sung, S. Baek, M. Barnes, J. Candy, A. Dominguez, D. Ernst, C. Gao, A. E. Hubbard, J. W. Hughes, Y. Lin, D. Mikkelsen, F. Parra, M. Porkolab, J. E. Rice, J. Walk, S. J. Wukitch, and Team Alcator C-Mod. Phys. Plasmas, 20(056106), 2013.

${ }^{16}$ A. E. White, N. T. Howard, A. J. Creely, M. A. Chilenski, M. Greenwald, A. E. Hubbard, J. W. Hughes, E. Marmar, J. E. Rice, J. M. Sierchio, C. Sung, J. R. Walk, D. G. Whyte, D. R. Mikkelsen, E. M. Edlund, C. Kung, C. Holland, J. Candy, C. C. Petty, M. L. Reinke, and C. Theiler. Nonlinear gyrokinetic simulations of the i-mode high confinement regime and comparisons with experimenta). Physics of Plasmas, 22(5), 2015.

${ }^{17}$ A. Casati, T. Gerbaud, P. Hennequin, C. Bourdelle, J. Candy, F. Clairet, X. Garbet, V. Grandgirard, ö. Gürcan, S. Heuraux, G. T. Hoang, C. Honoré, F. Imbeaux, R. Sabot, Y. Sarazin, L. Vermare, and R. E. Waltz. Phys. Rev. Lett, 102(165005), 2009.

${ }^{18}$ S. Leerink, V. V. Bulanin, A. D. Gurchenko, E. Z. Gusakov, J. A. Heikkinen, S. J. Janhunen, S. I. Lashkul, A. B. Altukhov, L. A. Esipov, M. Yu. Kantor, T. P. Kiviniemi, T. Korpilo, D. V. Kuprienko, and A. V. Petrov. Phys. Rev. Lett, 109(165001), 2012.

${ }^{19}$ F. Jenko, D. Told, T. Görler, J. Citrin, A. B. Navarro, C. Bourdelle, S. Brun- 
ner, G. Conway, T. Dannert, H. Doerk, D.R. Hatch, J.W. Haverkort, J. Hobirk, G.M.D. Hogeweij, P. Mantica, M.J. Pueschel, O. Sauter, L. Villard, E. Wolfrum, and the ASDEX Upgrade Team. Global and local gyrokinetic simulations of high-performance discharges in view of iter. Nuclear Fusion, 53(7):073003, 2013

${ }^{20}$ T. Happel, A. B. Navarro, G. D. Conway, C. Angioni, M. Bernert, M. Dunne, E. Fable, B. Geiger, T. Görler, F. Jenko, R. M. McDermott, F. Ryter, U. Stroth, and the ASDEX Upgrade Team. Phys. Plasmas, 22(032503), 2015

${ }^{21}$ A. B. Navarro, T. Happel, T. Görler, F. Jenko, J. Abiteboul, A. Bustos, H. Doerk, D. Told, and ASDEX Upgrade Team. Gyrokinetic studies of core turbulence features in asdex upgrade h-mode plasmas. Physics of Plasmas, 22(042513), 2015

${ }^{22}$ U. Stroth, A. B. Navarro, G.D. Conway, T. Görler, T. Happel, P. Hennequin, C. Lechte, P. Manz, P. Simon, A. Biancalani, E. Blanco, C. Bottereau, F. Clairet, S. Coda, T. Eibert, T. Estrada, A. Fasoli, L. Guimarais, Ö. Gürcan, Z. Huang, F. Jenko, W. Kasparek, C. Koenen, A. Krämer-Flecken, M.E. Manso, A. Medvedeva, D. Molina, V. Nikolaeva, B. Plaum, L. Porte, D. Prisiazhniuk, T. Ribeiro, B.D. Scott, U. Siart, A. Storelli, L. Vermare, and S. Wolf. Experimental turbulence studies for gyro-kinetic code validation using advanced microwave diagnostics. Nuclear Fusion, 55(8):083027, 2015.

${ }^{23}$ J. Abiteboul, T. Görler, F. Jenko, D. Told, and ASDEX Upgrade Team. Global electromagnetic simulations of the outer core of an asdex upgrade 1-mode plasma. Physics of Plasmas, 22(092314), 2015.

${ }^{24} \mathrm{~J}$. Candy and R. E. Waltz. Anomalous transport scaling in the diii-d tokamak matched by supercomputer simulation. Phys. Rev. Lett., 91:045001, Jul 2003.

${ }^{25}$ J. Candy, R. E. Waltz, and W. Dorland. The local limit of global gyrokinetic simulations. Physics of Plasmas, 11(5):L25-L28, 2004

${ }^{26}$ B. F. McMillan, X. Lapillonne, S. Brunner, L. Villard, S. Jolliet, A. Bottino, T. Görler, and F. Jenko. System size effects on gyrokinetic turbulence. Phys. Rev. Lett., 105:155001, Oct 2010.

${ }^{27}$ T. Görler, X. Lapillonne, S. Brunner, T. Dannert, F. Jenko, S.K. Aghdam, P. Marcus, B.F. McMillan, F. Merz, Ol. Sauter, D. Told, and L. Villard.
Flux- and gradient-driven global gyrokinetic simulation of tokamak turbulencea). Physics of Plasmas, 18(056103), 2011.

${ }^{28}$ F. Jenko, W. Dorland, M. Kotschenreuther, and B. N. Rogers. Phys. Plasmas, 7(1904), 2000.

${ }^{29}$ M. A. Beer, S. C. Cowley, and G. W. Hammett. Field aligned coordinates for nonlinear simulations of tokamak turbulence. Physics of Plasmas, 2(7):2687-2700, 1995.

${ }^{30}$ X. Garbet, Y. Sarazin, P. Beyer, P. Ghendrih, R.E. Waltz, M. Ottaviani, and S. Benkadda. Flux driven turbulence in tokamaks. Nuclear Fusion, 39(11Y):2063, 1999.

${ }^{31}$ Y. Sarazin, V. Grandgirard, J. Abiteboul, S. Allfrey, X. Garbet, Ph. Ghendrih, G. Latu, A. Strugarek, and G. Dif-Pradalier. Large scale dynamics in flux driven gyrokinetic turbulence. Nuclear Fusion, 50(5):054004, 2010.

${ }^{32}$ Y. Idomura, H. Urano, N. Aiba, and S. Tokuda. Study of ion turbulent transport and profile formations using global gyrokinetic full- $\mathrm{f}$ vlasov simulation. Nuclear Fusion, 49(6):065029, 2009.

${ }^{33}$ T. Görler, X. Lapillonne, S. Brunner, T. Dannert, F. Jenko, F. Merz, and D. Told. The global version of the gyrokinetic turbulence code GENE. Journal of Computational Physics, 230(18):7053 - 7071, 2011.

${ }^{34}$ B. F. McMillan, S. Jolliet, T. M. Tran, L. Villard, A. Bottino, and P. Angelino. Long global gyrokinetic simulations: Source terms and particle noise control. Physics of Plasmas, 15(052308), 2008.

${ }^{35}$ X. Lapillonne, B. F. McMillan, T. Görler, S. Brunner, T. Dannert, F. Jenko, F. Merz, and L. Villard. Nonlinear quasisteady state benchmark of global gyrokinetic codes. Physics of Plasmas, 17(112321), 2010.

${ }^{36}$ H. Doerk. Ph.D. thesis, Universität Ulm, 2013.

${ }^{37}$ P. Xanthopoulos, W. A. Cooper, F. Jenko, Yu. Turkin, A. Runov, and J. Geiger. Phys. Plasmas, 16(2303), 2009.

${ }^{38}$ M.J. Pueschel, T. Dannert, and F. Jenko. On the role of numerical dissipation in gyrokinetic vlasov simulations of plasma microturbulence. Computer Physics Communications, 181(8):1428 - 1437, 2010.

${ }^{39}$ G. Pereverzev and P. N. Yushmanov, Technical Report No. Ipp 5/98, MaxPlanck Institute (2002).

${ }^{40}$ J. C. Hillesheim, C. Holland, L. Schmitz, S. Kubota, T. L. Rhodes, and T. A. Carter. Review of Scientific Instruments, 83(10E331), 2012. 